\title{
Improved particle swarm optimization approach for nonconvex static and dynamic economic power dispatch
}

\author{
Rajkumari Batham ${ }^{1}$, Kalpana Jain $^{2}$ and Manjaree Pandit ${ }^{3 *}$ \\ * Department of Electrical Engineering, Madhav Institute of Technology and Science Gwalior, INDIA \\ "Corresponding Author: manjaree_p@hotmail.com, Tel +91-0751-2665962, +91-0751-2409380
}

\begin{abstract}
The cost of power generation in fossil fuel plants is very high and economic dispatch helps in saving a significant amount of revenue. For practical generators the economic dispatch problem gets translated into a complex non-convex, multimodal optimization problem which can not be solved by traditional gradient based optimization methods. The complexity further increases due to the multiple constraints that need to be satisfied. This paper proposes an improved particle swarm optimization approach (IPSO) for solving nonconvex static and dynamic economic dispatch. The classical PSO (CPSO) approach suffers from the problem of premature convergence, particularly for complex multimodal functions. The idea behind IPSO is i) to enhance the search capability of the CPSO by reinitializing the velocity vector whenever saturation sets in and ii) to use a parameter automation strategy to strike a proper balance between local and global search. The performance of IPSO has been tested on five standard test cases. The results are compared with previously published literature and are found to be comparable/or superior.
\end{abstract}

Keywords: Improved PSO, premature convergence, static/dynamic economic dispatch, prohibited operating zones, ramp rate limits, and valve point loading effects.

\section{Introduction}

Economic power dispatch is one of the most important functions of modern energy management system. This problem is formulated as a constrained optimization problem with the objective of generation allocation to the power generators to minimize total fuel cost with satisfaction of all operating constraints. Conventional methods usually assume the input-output characteristics of power generators, known as cost curves, to be quadratic or piecewise quadratic, monotonically increasing functions. But modern generating units have a variety of non-linearities in their cost curves due to valve point loading and other effects, which make this assumption inaccurate and the resulting approximate solutions cause a lot of revenue loss over time. On the other hand, evolutionary methods such as genetic algorithms (GA) and particle swarm optimization (PSO), differential evolution (DE) and bacterial foraging $(\mathrm{BF})$ are free from convexity assumptions and perform better due to their excellent parallel search capability. Therefore, these methods are particularly popular for solving non linear, nonconvex, discontinuous optimization problems. This paper focuses on solution of nonconvex ED problem using an improved PSO based method.

Conventional gradient based optimization methods like lamda iteration, base point participation factor, gradient methods etc. rely heavily on the convexity assumption of generator cost curves and hence approximate these curves using quadratic or piecewise quadratic monotonically increasing cost functions (Wood et al.,1984); resulting solutions are inaccurate and cause revenue losses. This assumption is not valid for practical generators because the cost functions of generators have discontinuities and higher order nonlinearities due to valve point loading (Walter et al., 1993, Sinha et al., 2003), prohibited operating zones (Orero et al., 1996) and ramp rate limits of generators (Wang et al., 1993). The practical ED with above nonlinearities translates into a complicated optimization problem having complex and nonconvex characteristics, with multiple minima, making the challenge of obtaining the global minima, very difficult. Dynamic programming (Shoults et al., 1986) has no restriction on the shape of cost curves, but this method is computationally extensive, time consuming and suffers from the problem of dimensionality. 
Methods like dynamic programming (Shoults et al., 1986), genetic algorithm (Walter et al., 1993, Sinha et al., 2003, Damousis et al., 2003), evolutionary programming (Sinha et al., 2003), and particle swarm optimization (Selvakumar et al., 2007, Chaturvedi et al., 2008, Park et al., 2007, Victoire et al., 2005, Park et al., 2010) solve nonconvex optimization problems efficiently and often achieve a fast and near global optimal solution. The PSO, which was first introduced by Kennedy and Eberhart (Kennedy, et al., 1995), is a powerful, robust, population based algorithm with inherent parallelism. This method is increasingly gaining acceptance for solving economic dispatch (Chaturvedi et al., 2008, Park et al., 2007, Victoire et al., 2005, Park et al., 2010) and a variety of power system problems, due to its simplicity, superior convergence characteristics and high solution quality. Recent research however has observed that PSO approach suffers from premature convergence, particularly for complex functions having multiple minima (Victoire et al., 2005, Park et al., 2010).

The most important issue with evolutionary techniques is to maintain a proper balance between exploration i.e. global search and exploitation i.e. local search. The performance of evolutionary methods heavily depends on the settings of the tuning parameters; therefore finding optimal parameter setting is a very big challenge. Evolutionary methods also have a tendency to converge very fast to a solution that is quite close to the global minimum. This tendency causes premature convergence. Researchers employ various parameter automation strategies and hybridization of global and local optimization techniques. In reference (Park et al., 2010) performance improvement for nonconvex economic dispatch problem was reported by integrating the PSO with chaotic sequences and crossover operation. The concept of variable scaling factor based on the one-fifth success rule of evolutionary strategies is employed in reference (Chiou et al., 2009). Some researchers have proposed hybrid methods combining the advantages of two evolutionary methods to get improved performance (Bhattacharya et al., 2010).It has been observed by various researchers that the classical PSO (CPSO) very quickly finds a good local solution but gets stuck there for a number of iterations without further improvement. As a result, it becomes tedious to find global best solutions for complicated nonconvex problems having multiple local minima and an irregular search space.

To handle the problem of premature convergence, an improved PSO (IPSO) algorithm is proposed in this paper. The improved PSO employs a two-tier approach to avoid saturation and premature convergence. The improved PSO (IPSO) i) introduces crazy particles with randomized velocities to maintain momentum in the search and to avoid saturation ii) employs a novel parameter automation strategy in which the cognitive and social parameters are dynamically tuned in order to efficiently control the local and global search in such a manner that global convergence is achieved. The performance of the improved PSO (IPSO) is significantly better than the classical PSO (CPSO).

The performance of the proposed IPSO has been tested on a standard test system having prohibited operating zones and ramp rate limits. Five test cases with different complexity levels have been taken. It is observed that the IPSO approach outperforms CPSO. Results are compared with recently published literature (Bhattacharya et al, 2010, Panigrahi et al, 2008, Chen et al, 1995, Naresh et al., 2004) and superiority of IPSO has been established.

\section{Nonconvex Economic Dispatch Formulation}

The practical static and dynamic NCED problem with generator nonlinearities such as valve point loading effects, prohibited operating zones and ramp rate limits, are solved in this paper using IPSO based approach to find the optimal generation dispatch for different operating conditions.

2.1 Valve point loading effects: The valve-point effects introduce ripples in the heat-rate curves and make the objective function discontinuous, nonconvex and with multiple minima. For accurate modeling of valve point loading effects, a rectified sinusoidal function (Walter et al, 1993) is added in the cost function in this Paper. The fuel input-power output cost function of ith unit is given as

$F_{i}\left(P_{i}\right)=a_{i} P_{i}^{2}+b_{i} P_{i}+c_{i}+\left|e_{i} \times \sin \left(f_{i} \times\left(P_{\min }-P_{i}\right)\right)\right|$

where $a_{i}, b_{i}$ and $c_{i}$ are the fuel-cost coefficients of the $i^{\text {th }}$ unit, and $e_{i}$ and $f_{i}$ are the fuel cost-coefficients of the $i^{\text {th }}$ unit with valve-point effects. The NCED problem is to determine the generated powers Pi of units for a total load of PD so that the total fuel cost, $F_{T}$ for the N number of generating units is minimized subject to the power balance constraint and unit upper and lower operating limits. The objective is

$$
\begin{aligned}
& \operatorname{Min} F_{T}=\sum_{i=1}^{N} F_{i}\left(P_{i}\right) ; \text { Subject to the constraints given by } \\
& \sum_{i=1}^{N} P_{i}-\left(P_{D}+P_{L}\right)=0
\end{aligned}
$$




$$
P_{i}^{\min } \leq P_{i} \leq P_{i}^{\max } \quad i=1,2, \ldots, N
$$

For a given total real load PD the system loss PL is a function of active power generation at each generating unit. To calculate system losses, methods based on penalty factors and constant loss formula coefficients or B-coefficients (Wood et al,1984) are in use. The latter is adopted in this paper as per which transmission losses are expressed as

$$
P_{L}=\sum_{i=1}^{N} \sum_{j=1}^{N} P_{i} B_{i j} P_{j}+\sum_{i=1}^{N} B_{o i} P_{i}+B_{o o}
$$

For further details, (Wood et al., 1984) may be referred.

2.2 Generator ramp rate limits: When the generator ramp rate limits are considered, the operating limits are modified as follows:

$\operatorname{Max}\left(P_{i}^{\min }, P_{i}^{o}-D R_{i}\right) \leq P_{i} \leq \operatorname{Min}\left(P_{i}^{\max }, P_{i}^{o}+U R_{i}\right)$

The previous operating point of $\mathrm{i}^{\text {th }}$ generator is $P_{i}^{o}$ and $\mathrm{DR}_{\mathrm{i}}$ and $\mathrm{UR}_{\mathrm{i}}$ are the down and up ramp rate limits respectively.

2.3 Prohibited operating zones: The cost curves of practical generators are discontinuous as whole of the unit operating range is not always available for allocation. In other words, the generating units have prohibited operating zones due to some faults in the machines or their accessories such as pumps or boilers etc. (Orero et al, 1996). A unit with prohibited operating zones has discontinuous input-output characteristics. This feature can be included in the NCED formulation as follows:

$$
P_{i} \in\left\{\begin{array}{l}
P_{i}^{\min } \leq P_{i} \leq P_{i 1}^{{ }^{L}} \\
P_{i k-1}^{U} \leq P_{i} \leq P_{i k}^{{ }^{L}} \\
P_{i z i}^{U} \leq P_{i} \leq P_{i}{ }^{\max }
\end{array}\right.
$$

Here $\mathrm{z}_{\mathrm{i}}$ are the number of prohibited zones in $\mathrm{i}^{\text {th }}$ generator curve, $\mathrm{k}$ is the index of prohibited zone of $\mathrm{i}^{\text {th }}$ generator, $P_{i k}{ }^{L}$ is the lower limit of $\mathrm{k}^{\text {th }}$ prohibited zone, and $P_{i k-1}^{U}$ is the upper limit of $\mathrm{k}^{\text {th }}$ prohibited zone of $\mathrm{i}^{\text {th }}$ generator

2.4 Nonconvex dynamic economic load dispatch(NCDED): Dynamic Economic Load dispatch deals with sharing the system load including system losses among the available generators in such a way that all equality and inequality constraints are met and the cost of operation is minimized for each time interval.

In order to solve dynamic load dispatch problem, ramp-rate limit must be considered. The dynamic economic load dispatch (DELD) model can be described as follows: -

$$
\left\{\begin{array}{c}
\min f(t)=\sum_{i=1}^{N} F\left(P_{i}(t)\right) \\
\sum_{i=1}^{N} P_{i}(t)=P_{D}(t)+P_{L}(t) \\
P_{i \text { min }} \leq P_{i}(t) \leq P_{i \max } \\
-D R_{i} \leq P_{i}(t)-P_{i}(t-1) \leq U R_{i}
\end{array}\right.
$$

Where $\mathrm{N}$ is total numbers of committed units; $\mathrm{P}_{\mathrm{i}}(\mathrm{t})$ is active power output of the $\mathrm{i}^{\text {th }}$ unit at time $t$. The fuel cost function $\mathrm{f}(\mathrm{t})$ is given by

$$
f(t)=\sum_{i=1}^{N} F\left(P_{i}(t)\right)=\sum_{i=1}^{N}\left(a_{i}+b_{i} P_{i}(t)+c_{i} P_{i}^{2}(t)\right)+\mid e_{i} \times \operatorname{Sin}\left\{f_{i} \times\left(P_{i \min }-P_{i}(t)\right\} \mid\right.
$$

Here $a_{i}, b_{i}$ and $c_{i}$ are cost coefficients, $\mathrm{Pi}$ min is minimum output, $\mathrm{DR}_{\mathrm{i}}$ is down ramp rate limit, $\mathrm{UR}_{\mathrm{i}}$ is up ramp rate limit of the $\mathrm{i}^{\text {th }}$ unit; $\mathrm{P}_{\mathrm{D}}(\mathrm{t})$ is load demand at time interval $\mathrm{t} ; \mathrm{P}_{\mathrm{L}}(\mathrm{t})$ is network loss at time interval $\mathrm{t}$. 


\section{Particle Swarm Optimization}

Various conventional as well as modern methods have been employed for solving the non-linear, non-convex, discontinuous economic dispatch problem. The particle swarm optimization method has become quite popular for solving complex problems during the last couple of years. Its excellent random parallel search capability and constraint handling mechanism make it very efficient for locating good solution in the complex search domain.

3.1 Classical Particle swarm optimization (CPSO): The PSO (Kennedy, et al., 1995) is a population based modern heuristic search method inspired by the movement of a flock of birds searching for food. It is a simple and powerful optimization tool which scatters random particles i.e. solutions into the problem space. These particles, called swarms collect information from each other through an array constructed by their respective positions. The particles update their positions using the velocity of particles. Position and velocity are both updated in a heuristic manner using guidance from a particle's own experience and the experience of its neighbors.

The position and velocity vectors of the ith particle of a d-dimensional search space can be represented as $X_{i}=\left(x_{i 1}, x_{i 2}, \ldots \ldots \ldots \ldots x_{i d}\right)$ and $V_{i}=\left(v_{i 1}, v_{i 2}, \ldots \ldots . . v_{i d}\right)$ respectively. On the basis of the value of the evaluation function, the best previous position of a particle is recorded and represented as $p b e s t_{i}=\left(p_{i 1}, p_{i 2} \ldots \ldots . . . p_{i d}\right)$. The particle tries to modify its position using the current velocity and the distance from pbest and gbest. The modified velocity and position of each particle for fitness evaluation in the next iteration are calculated using the following equations (Kennedy, et al., 1995):

$$
\begin{aligned}
& v_{i d}^{k+1}=C\left[w^{*} v_{i d}^{k}+c_{1} * \text { rand }_{1} *\left(\text { pbest }_{i d}-x_{i d}\right)+c_{2} * \text { rand }_{2} *\left(\text { gbest }_{g d}-x_{i d}\right)\right] \\
& x_{i d}^{k+1}=x_{i d}+v_{i d}^{k+1}
\end{aligned}
$$

Here $\mathrm{w}$ is the inertia weight parameter which controls the global and local exploration capabilities of the particle. Constant $\mathrm{C}$ is constriction factor, $C_{1}, C_{2}$ are cognitive and social coefficients, and rand $_{1}$, rand ${ }_{2}$ are random numbers between 0 and 1 . A larger inertia weight factor is used during initial exploration and its value is gradually reduced as the search proceeds. The timevarying inertial weight is given by (Eberhart et al., 1999).

$$
w=\left(w_{\max }-w_{\min }\right) \times \frac{\left(\text { iter }_{\max }-\text { iter }\right)}{\text { iter }_{\max }}+w_{\text {min }}
$$

where iter $_{\max }$ is the maximum number of iterations. Constant $\mathrm{c}_{1}$ pulls the particles towards local best position whereas $\mathrm{c}_{2}$ pulls it towards the global best position. Usually these parameters are selected in the range of 0 to 4 . To improve the convergence of PSO algorithm, the constriction factor $C$ is also used. (Eberhart et al, 1999).For further details, (Kennedy et al., 1995; Eberhart et al., 1999) may be referred.

$$
C=\frac{2}{\left|2-\varphi-\sqrt{\varphi^{2}-4 \varphi}\right|} \quad \text { where } 4.1 \leq \varphi \leq 4.2
$$

As $\varphi$ increases, the factor $\mathrm{C}$ decreases and convergence becomes slower because population diversity is reduced. The value of $\mathrm{C}$ is also decreased iteratively similar to $\mathrm{w}$.

3.2 Improved PSO: The proposed IPSO employs a two-tier strategy to combat premature convergence phenomenon in PSO. By the combined use of i) crazy particles and ii) time varying acceleration coefficients (TVAC) it is ensured that a) population diversity is maintained and b) A proper balance is maintained between exploration (global search) and exploitation(local search). The idea behind using crazy particles is to randomize the velocities of some of the particles, (referred to as "crazy particles"), selected by applying a certain probability. In (Victoire et al, 2005), the probability of craziness $\rho_{c r}$ is defined as a function of inertia weight,

$$
\rho_{c r}=w_{\min }-\exp \left(-\frac{w^{k}}{w_{\max }}\right)
$$

Then velocities of particles are randomized as per the following logic: 
$v_{j}^{k}=\left\{\begin{array}{l}\operatorname{rand}\left(o, v_{\max }\right) ; \text { if } \rho_{c r} \geq \operatorname{rand}(0,1) \\ v_{j}^{k}, \text { otherwise }\end{array}\right.$

If the PSO algorithm tends to saturate in the beginning then a high value of $\rho_{c r}$ is used to create crazy particles, and a comparatively lower value is used at later stages of search.

The time-varying inertia weight (TVIW) can locate good solutions at a significantly faster rate but its ability to fine tune the optimum solution is weak, due to the lack of diversity at the end of the search. It has been observed by most researchers that in PSO, problem-based tuning of parameters is a key factor to find the optimum solution accurately and efficiently. Kennedy and Eberhart (Kennedy et al, 1995) stated that a relatively higher value of the cognitive component, compared with the social component, results in roaming of individuals through a wide search space. On the other hand, a relatively high value of the social component leads particles to a local optimum prematurely. Normally studies keep each of the acceleration coefficients at 2 , in order to make the mean of both stochastic factors in (7) equal to one, so that particles would over fly only half the time of search.

In population-based optimization methods, the policy is to encourage the individuals to roam through the entire search space, during the initial part of the search, without clustering around local optima. During the latter stages, however convergence towards the global optima should be encouraged, to find the optimum solution efficiently. In TVAC, this is achieved by changing the acceleration coefficients c1 and c2 with time in such a manner that the cognitive component is reduced while the social component is increased as the search proceeds. A large cognitive component and small social component at the beginning, allows particles to move around the search space, instead of moving towards the population best prematurely. During the latter stage in optimization, a small cognitive component and a large social component allow the particles to converge to the global optima. The acceleration coefficients are expressed as (Ratnaweera et al., 2004):

$$
\begin{aligned}
& c_{1}=\left(c_{1 f}-c_{1 i}\right) \frac{\text { iter }}{\text { iter }_{\text {max }}}+c_{1 i} \\
& c_{2}=\left(c_{2 f}-c_{2 i}\right) \frac{\text { iter }_{\text {iter }}}{\text { max }}+c_{2 i}
\end{aligned}
$$

The velocity is calculated by substituting eq. (14) and (15) in eq. (7) to get

$$
\begin{aligned}
& v_{i d}^{k+1}=C\left[w \times v_{i d}^{k}+\left(\left(c_{i f}-c_{1 i}\right) \frac{\text { iter }}{\text { iter }_{\max }}+c_{1 i}\right) \times \text { rand }_{1} \times\left(\text { pbest }_{i d}-x_{i d}\right)+\left(\left(c_{2 f}-c_{2 i}\right) \frac{\text { iter }}{\text { iter }_{\max }}+c_{2 i}\right)\right. \\
& \left.\times \text { rand }_{2} \times\left(\text { gbest }_{g d}-x_{i d}\right)\right]
\end{aligned}
$$

where $c_{1 i}, c_{1 f}, c_{2 i}$ and $c_{2 f}$ are initial and final values of cognitive and social acceleration factors respectively.

\section{Implementation of IPSO for static/dynamic ED solution}

The step by step procedure for implementation of the IPSO algorithm for solving static and dynamic economic dispatch problem with valve point loading effect prohibited operating zones and ramp rate limits is given below. The flowchart for this algorithm is given in Figure. 1.

\subsection{Solution of constrained static and dynamic nonconvex economic dispatch using IPSO}

Most of the PSO algorithms suffer from the problem of premature convergence in the early stages of the search and henceforth are unable to locate the global optimum (Selvakumar et al., 2007, Chaturvedi et al., 2008, Park et al., 2007, Victoire et al., 2005, Park et al., 2010). The proposed IPSO algorithm employs a two-tier strategy to improve the performance of classical PSO. First, the crazy particles, whose probability can be controlled, do not allow saturation to set in. The idea is to randomize the velocities to maintain momentum in the optimization process and improve the solution quality. Secondly, the cognitive and social acceleration coefficients are iteratively controlled to keep a balance between global and local search. The IPSO algorithm achieves significantly better results as compared to the classical PSO. The implementation consists of the following steps.

Step 1- Initialization of the swarm: For a population size $P$, the particles are randomly generated in the range 0-1 and located between the maximum and the minimum operating limits of the generators. If there are $N$ generating units, the $i^{\text {th }}$ particle is 
represented as $P_{i}=\left(P_{i 1}, P_{i 2}, P_{i 3} \ldots \ldots \ldots \ldots \ldots \ldots \ldots P_{i N}\right) . \quad$ The $\mathrm{j}^{\text {th }}$ dimension if the $\mathrm{i}^{\text {th }}$ particle is allocated a value of $P_{i j}$ as given below to satisfy the constraint given by eq. (3) Here, $r$ is a random number, $r \in[0,1]$.

$P_{i j}=P_{j \min }+r\left(P_{j \max }-P_{j \min }\right)$

For generators with ramp rate limits, the initialization is based on eq. (5). For limiting the operation within the prohibited zones, the particles are clamped at their respective lower or upper zone limits, (whichever is nearer to the particle position) as per eq. (6).

Step 2- Evaluation of swarm population: The merit of each individual particle in the swarm is found using a fitness function called evaluation function. The evaluation function should be such that cost is minimized while constraints are satisfied. The popular penalty function method employs functions composed of squared or absolute violations to reduce the fitness of the particle in proportion to the magnitude of the violation. Large values for penalty parameters ill condition the penalty function while very small values do not allow the violations to contribute effectively in penalizing a particle. Therefore, the penalty parameters are chosen carefully to distinguish between feasible and infeasible solution. Hence, the penalty parameters, are chosen such that an infeasible solution is awarded fitness worse than the weakest feasible string. Since two infeasible strings are not treated equally, the string further away from the feasibility boundary, is more heavily penalized. Thus, a constrained optimization problem is converted to unconstrained optimization problem.

The evaluation function $f\left(P_{i}\right)$ is defined to minimize the non smooth cost function given by eq.(1) for a given load demand $P_{D}$ while satisfying the constraints given by eq. $(2,3)$ as:

$f\left(P_{i}\right)=\sum_{i=1}^{N} F_{i}\left(P_{i}\right)+\alpha\left[\sum_{i=1}^{N} P_{i}-\left(P_{D}+P_{L}\right)\right]^{2}+\beta\left[\sum_{k=1}^{n_{i}} P_{i}(\text { violation })_{k}\right]^{2}$

where $\alpha$ is the penalty parameter for not satisfying load demand and $\beta$ represents the penalty for a unit loading falling within a prohibited operating zone.

Step-3: Initialization of pbest and gbest: The fitness values obtained above for the initial particles of the swarm are set as the initial pbest values of the particles. The best value among all the pbest values is identified as gbest.

Step- 4: Generation of crazy particles: To control excessive roaming of particles, velocity is restricted between $-V_{j}^{\max }$ and $+V_{j}^{\max }$.Here, $\mathrm{R}$ is selected such that $V_{j}{ }^{\max }$ lies between $15-20 \%$ of the range of the variable. The maximum velocity limit for the

$j^{\text {th }}$ generating unit is computed as: $V_{j}^{\max }=\frac{P_{j . \max }-P_{j \min }}{R}$

The velocity vector is randomized using eq. (11) and (12).

Step-5: Updating the swarm: The particle position vector is updated using eq. (16). The values of the evaluation function are calculated for the updated positions of the particles. If the new value is better than the previous pbest, the new value is set to pbest. Similarly, value of gbest is also updated if the best pbest is better than the stored value of gbest.

Step- 6: Stopping criteria: A stochastic optimization algorithm is stopped either based on the tolerance limit or maximum number of iterations. In this Paper maximum number of iterations is adapted as the stopping criterion after which the positions of gbest are stored as the optimal solution.

\section{Test Results and Analysis}

A novel improved PSO (IPSO) algorithm is proposed in which a dual strategy is employed to avoid saturation and premature convergence of the population, particularly for complex functions. The idea here is i) to exercise proper control over the global and local exploration of the swarm during the optimization process by using TVAC ii) to reinitialize the velocity vector whenever it stagnates causing saturation.

The performance of the IPSO is compared here with the classical PSO. It is observed that all the IPSO performs significantly better that the classical PSO for complex functions. Simulations were carried out using MATLAB 7.0.1 on a Pentium IV processor, $2.8 \mathrm{GHz}$. with $1 \mathrm{~GB}$ RAM.

\subsection{Description of test systems}

A system with three thermal generating units (Wood et al, 1984, Chen et al, 1995) is used to demonstrate the performance of the proposed IPSO algorithm. The system cost coefficients and other data is given in the Appendix section. The system has many 
complexities and constraints such as i) Valve point loading (VPL) ii) Prohibited operating zone (POZ)and iii) Transmission losses in addition to the generating capacity constraints and power balance equation. The optimal solutions are computed under five different conditions; the first three test cases are solved for static economic dispatch while the last two test cases are solved for dynamic economic dispatch.

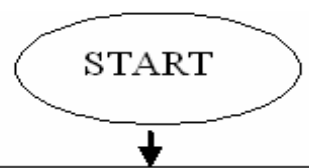

Create a random initial population of feasible $P_{j}$ values between generator limits using eq. ( $3 \& 18$ ) and POZ using eq. (6). Read initial schedule $P^{\circ}$, Apply RRL using eq. (5)

Evaluate initial fitness using (19); Initialize pbest, gbest, velocity, iteration count, set initial and final acceleration coefficients

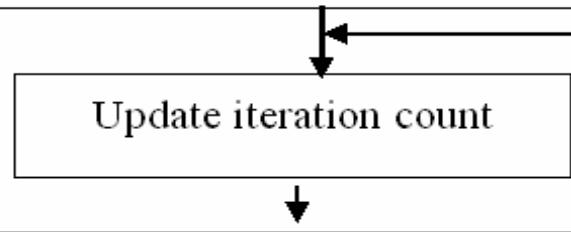

Update position and velocity using eq. (10) and (17); generate crazy particles with eq. $(13,14)$; Apply velocity limits given by eq. (20)

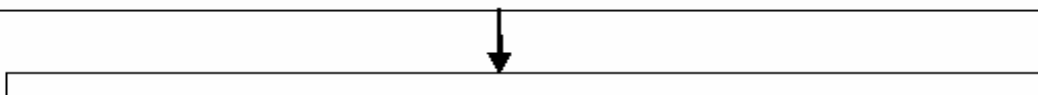

Check for $\mathrm{P}_{\min }, \mathrm{P}_{\max }$, and POZ limits; apply correction if needed ;Evaluate penalized cost using eq. (19)

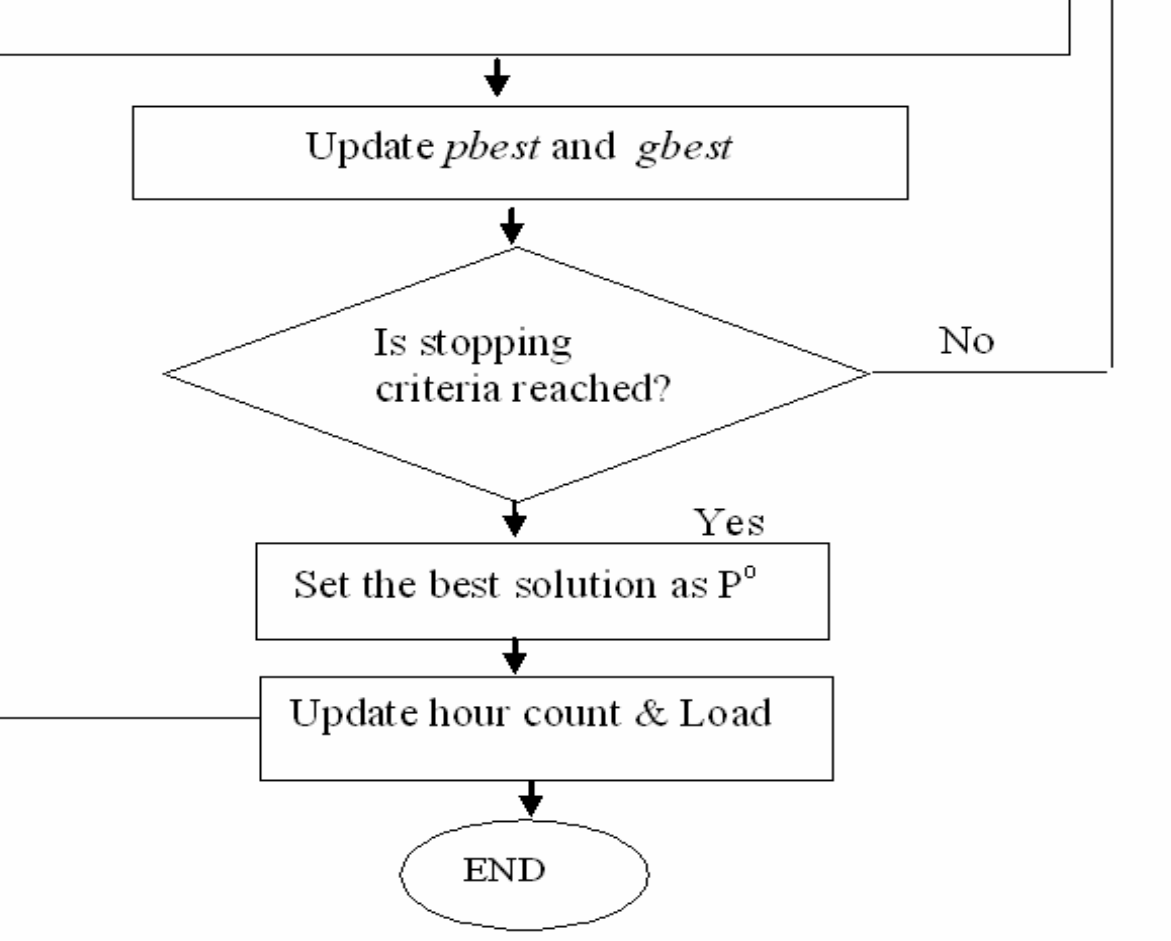

Figure 1. Flowchart of Nonconvex Dynamic Economic Dispatch (NCDED) using IPSO method 
Test Case I: The above system with RRL and POZ but without VPL and losses

Test Case II: The above system with RRL, POZ and losses but without VPL

Test Case III: The above system with RRL, POZ and VPL but without losses

Test Case IV: The dynamic 24-hour scheduling with POZ and RRL

Test Case V: The dynamic 24-hour scheduling with POZ, VPL and RRL

For Test Case II the IPSO results are compared and validated with previously published results (Bhattacharya et al, 2010). The IPSO has achieved better results than those reported in literature (Bhattacharya et al, 2010).The Performance of IPSO is also compared with classical PSO (CPSO) method in terms of convergence behavior, solution quality, consistency and computational efficiency and it was observed that IPSO outperforms CPSO. The superiority of IPSO over CPSO in more pronounced for complex Test Cases II (with loss) and III (with VPL).

\subsection{Parameter set-up}

The number of iterations used is $100, w$ is varied from 0.9 to 0.4 using (9), constriction factor is also reduced from 0.73 to 0.64 similar to the inertial weight $\mathrm{w}$ (for, $4.1 \leq \varphi \leq 4.2$ in eq. 12) as search proceeds. These values The value of $\mathrm{c}_{1}=\mathrm{c}_{2}=2.0$ is found to be most suitable for CPSO. Population size was set at 100 for all the five test cases.

\subsection{Effect of cognitive and social coefficients}

To evaluate the role of TVAC in solving complex DED problem, the values of $c_{1 f}$ and $c_{2 i}$ were varied between 0.1 and 0.5 and it was noticed that the best results were obtained when both were fixed at 0.2 . Then the value of $c_{1 i}$ was increased from 1.8 to 2.5 and $c_{2 f}$ was reduced from 2.5 to 1.8 , while $c_{1 f}$ and $c_{2 i}$ were kept fixed at 0.2 . The initial value of cognitive parameter $c_{1 i}$ and final value of global parameter $c_{2 f}$ were targeted for study because $c_{1 i}$ controls the initial roaming of the swarm and $c_{2 f}$ facilitates global convergence in the final stage of the search. The final and initial values of $c_{1}$ and $c_{2}$ are to be selected by the user. The upper and lower limits for these values are normally 2.5 and 0.1 . To show the effect of this variation results were computed and tabulated in Table 1 for different parameter settings. The optimal values are found to be problem dependant. However, for every parameter setting the global minimum value is achieved, only the consistency of achieving the minima is different for different settings.

Table 1 shows the results of this variation on the minimum, maximum, average costs and their standard deviation (S.D) out of 50 different trials for Test Case I. Best results were obtained when $c_{1 i=} 2.5, c_{1 f}=0.2, c_{2 i}=0.2$ and $c_{2 f}=2.2$.

\subsection{Effect of population size}

The study carried out in this paper found that population size should be optimum for achieving global best results. Too large or a very small population may not be capable of searching a minimum, particularly in complex multimodal problems.

The population size is a very important issue in stochastic search methods. Too large a population makes an algorithm slow and computationally inefficient, while a very small population may not be capable of searching a minimum, particularly in complex problems. The optimum population size is found to be related to the problem dimension. Table 2 lists the performance of Test Case I for a population of 10, 20, 40, 60, 80 and 100. It can be seen that with increase in population, there is a steady improvement in minimum value, average value and S.D.

\subsection{Convergence Characteristics}

The convergence behavior of the CPSO and IPSO was compared employing the same evaluation function, same initial population and velocity for same number of iterations. The results for one trial are shown in Figure. 2. It can be seen that the IPSO exhibits superior convergence characteristics, i.e. saturation does not occur in the initial iterations.

\subsection{Solution Quality}

The dynamic convergence behavior of the IPSO and CPSO was also studied by calculating the mean and standard deviation of each individual in the swarm after each iteration. The mean value $\mu$ and standard deviation $\sigma$ are defined as:

$$
\mu=\frac{\sum_{i=1}^{P S} f\left(P_{i}\right)}{P S}
$$




$$
\sigma=\sqrt{\frac{1}{P S} \sum_{i=1}^{P S}}\left(f\left(P_{i}\right)-\mu\right)^{2}
$$

PS is the population size here and $f\left(P_{i}\right)$ is the evaluation function defined in (18). Figure 3 and Figure 4 plot and compare the standard deviation and mean of IPSO and PSO for Test Case I. The IPSO method clearly establishes its superiority over the classical PSO (CPSO) and produces better dynamic convergence, because the mean cost and the standard deviation of the swarm reduce continuously. The CPSO shows premature convergence and does not achieve minima for the complex 3-unit test case with RRL, POZ.

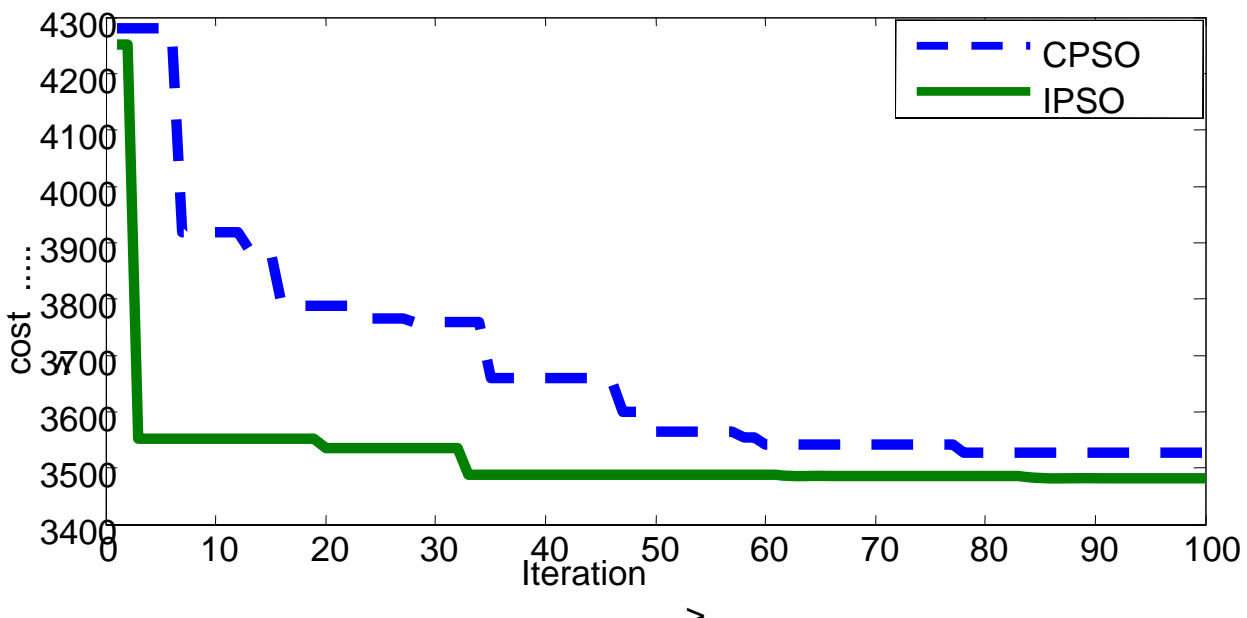

Figure 2. Comparison convergence characteristics

Table 1. Effect of acceleration coefficients on performance of IPSO (Test Case I; 50 trials)

\begin{tabular}{|c|c|c|c|c|c|c|c|c|}
\hline S.No. & $c_{1 i}$ & $c_{1 f}$ & $c_{2 i}$ & $c_{2 f}$ & $\begin{array}{l}\text { Minimum } \\
\text { Cost(\$/h) }\end{array}$ & $\operatorname{Max} \operatorname{cost}(\$ / h)$ & $\begin{array}{l}\text { Average } \\
\operatorname{cost}(\$ / h)\end{array}$ & S.D. \\
\hline 1 & \multirow{4}{*}{2.5} & 0.2 & 0.2 & 2.5 & $3.4829 \mathrm{e}+003$ & $3.5155 \mathrm{e}+003$ & $3.4892 \mathrm{e}+003$ & 4.4985 \\
\hline 2 & & 0.2 & 0.2 & 2.2 & $3.4829 e+003$ & $3.4834 e+003$ & $3.4887 e+003$ & 0.7362 \\
\hline 3 & & 0.2 & 0.2 & 1.9 & $3.4829 \mathrm{e}+003$ & $3.5156 \mathrm{e}+003$ & $3.4890 \mathrm{e}+003$ & 5.0013 \\
\hline 4 & & 0.2 & 0.2 & 1.8 & $3.4829 \mathrm{e}+003$ & $3.5177 \mathrm{e}+003$ & $3.4892 \mathrm{e}+003$ & 6.1077 \\
\hline 5 & \multirow{4}{*}{2.2} & 0.2 & 0.2 & 2.5 & $3.4829 \mathrm{e}+003$ & $3.5130 \mathrm{e}+003$ & $3.4904 \mathrm{e}+003$ & 5.9825 \\
\hline 6 & & 0.2 & 0.2 & 2.2 & $3.4829 \mathrm{e}+003$ & $3.5227 \mathrm{e}+003$ & $3.4902 \mathrm{e}+003$ & 6.4204 \\
\hline 7 & & 0.2 & 0.2 & 1.9 & $3.4830 \mathrm{e}+003$ & $3.5112 \mathrm{e}+003$ & $3.4883 \mathrm{e}+003$ & 4.3430 \\
\hline 8 & & 0.2 & 0.2 & 1.8 & $3.4829 \mathrm{e}+003$ & $3.5136 \mathrm{e}+003$ & $3.4887 \mathrm{e}+003$ & 4.8034 \\
\hline 9 & \multirow{4}{*}{2} & 0.2 & 0.2 & 2.5 & $3.4829 \mathrm{e}+003$ & $3.5161 \mathrm{e}+003$ & $3.4892 \mathrm{e}+003$ & 5.0779 \\
\hline 10 & & 0.2 & 0.2 & 2.2 & $3.4829 e+003$ & $3.5222 \mathrm{e}+003$ & $3.4892 \mathrm{e}+003$ & 5.0828 \\
\hline 11 & & 0.2 & 0.2 & 1.9 & $3.4829 \mathrm{e}+003$ & $3.5137 \mathrm{e}+003$ & $3.4894 \mathrm{e}+003$ & 5.6721 \\
\hline 12 & & 0.2 & 0.2 & 1.8 & $3.4829 \mathrm{e}+003$ & $3.5220 \mathrm{e}+003$ & $3.4907 \mathrm{e}+003$ & 6.5701 \\
\hline 13 & \multirow{4}{*}{1.8} & 0.2 & 0.2 & 2.5 & $3.4829 \mathrm{e}+003$ & $3.5093 e+003$ & $3.4892 \mathrm{e}+003$ & 4.9600 \\
\hline 14 & & 0.2 & 0.2 & 2.2 & $3.4829 e+003$ & $3.5216 \mathrm{e}+003$ & $3.4904 \mathrm{e}+003$ & 6.4442 \\
\hline 15 & & 0.2 & 0.2 & 1.9 & $3.4830 \mathrm{e}+003$ & $3.5046 \mathrm{e}+003$ & $3.4877 \mathrm{e}+003$ & 3.3622 \\
\hline 16 & & 0.2 & 0.2 & 1.8 & $3.4829 \mathrm{e}+003$ & $3.5195 e+003$ & $3.4896 \mathrm{e}+003$ & 5.8175 \\
\hline
\end{tabular}


Table 2. Effect of population size on performance of IPSO (Test Case I; 50 trials)

\begin{tabular}{|c|c|c|c|c|c|}
\hline $\begin{array}{c}\text { Population } \\
\text { Size }\end{array}$ & $\begin{array}{c}\text { Minimum } \\
\text { Cost }(\$ / \mathrm{h})\end{array}$ & Mean Cost $(\$ / \mathrm{h})$ & $\begin{array}{c}\text { Maximum } \\
\text { Cost }(\$ / \mathrm{h})\end{array}$ & $\begin{array}{c}\text { S.D. } \\
(50 \text { trials })\end{array}$ \\
\hline 10 & $3.4830 \mathrm{e}+003$ & $3.4924 \mathrm{e}+003$ & $3.5219 \mathrm{e}+003$ & 6.4093 & 1.368170 \\
\hline 20 & $3.4830 \mathrm{e}+003$ & $3.4915 \mathrm{e}+003$ & $3.5160 \mathrm{e}+003$ & 6.2054 & 2.040088 \\
\hline 40 & $3.4830 \mathrm{e}+003$ & $3.4909 \mathrm{e}+003$ & $3.5252 \mathrm{e}+003$ & 6.0338 & 2.785883 \\
\hline 60 & $3.4831 \mathrm{e}+003$ & $3.4887 \mathrm{e}+003$ & $3.5175 \mathrm{e}+003$ & 4.3751 & 3.791913 \\
\hline 80 & $3.4829 \mathrm{e}+003$ & $3.4848 \mathrm{e}+003$ & $3.4949 \mathrm{e}+003$ & 2.0369 & 5.625992 \\
\hline $\mathbf{1 0 0}$ & $\mathbf{3 . 4 8 2 9 e + 0 0 3}$ & $\mathbf{3 . 4 8 3 4 e + 0 0 3}$ & $\mathbf{3 . 4 8 8 7 e + 0 0 3}$ & $\mathbf{0 . 7 3 6 2}$ & $\mathbf{7 . 4 7 2 0 2 4}$ \\
\hline
\end{tabular}

\subsection{Comparison of best results}

It can be seen from Table 3 and Table 4 that the proposed IPSO achieves very good results as compared to the classical PSO. Table 3 presents the results for Test case I for different loads. The CPSO and IPSO achieve comparable results for this case with IPSO showing slight superiority. Both methods satisfy the constraints imposed by ramp rate limits (RRL), prohibited operating zones (POZ), power balance constraint and unit operating limits.

From Table 4 it can be seen that though the solution given by (Bhattacharya et al, 2010) is minimum, it is violating the ramp rate limits. On the other hand, IPSO satisfies all constraints without violations. As per the data given in Appendix section $\mathrm{P}^{0}=[215,72$, 98,] down ramp rate $\mathrm{DR}_{\mathrm{i}}=$ [95 7864$]$ and $\mathrm{P}_{\min }=[50,5,15]$. Due to ramp rate limits (RRL)the unit operating limits get modified as per eq. (5) and now unit $P_{\min (m o d i f i e d)}=\left[120,5\right.$, 34]. The solution given by (Bhattacharya et al, 2010) has $P_{3}=15$ which is infeasible as the lower limit for unit three becomes 34 due to RRL.

Table 5 gives the results of Test Case III (with VPL) for different loads. The cost function is plotted for generating unit 1 and 2 and shown in Figure 5(a) and Figure 5(b). In Figure 5(a) the cost curves with VPL effects are compared with quadratic cost curves for both units. The cost function becomes rippled due to VPL and discontinuous due to prohibited operating zones. In Figure 5(b) the effect of ramp rate limits (RRL) on the cost curves can be seen. The Pmin and Pmax limits get modified due to RRL. The resulting cost function is nonconvex and hence very difficult to optimize. Similar characteristics can also be drawn for P3 using the data $\left(\mathrm{P}_{\min }, \mathrm{P}_{\max }, \mathrm{POZ}\right.$ and cost coefficients) in the Appendix. Due to space shortage and to show the characteristics clearly, only two generator cost characteristics have been shown here.

For this complex non-convex system, the IPSO searches the global minimum value very effectively, where as the CPSO is not able to achieve the global best value due to the inclusion of valve point loading (VPL) effects. Thus, the superiority of the IPSO over CPSO is more prominent for complex cases.

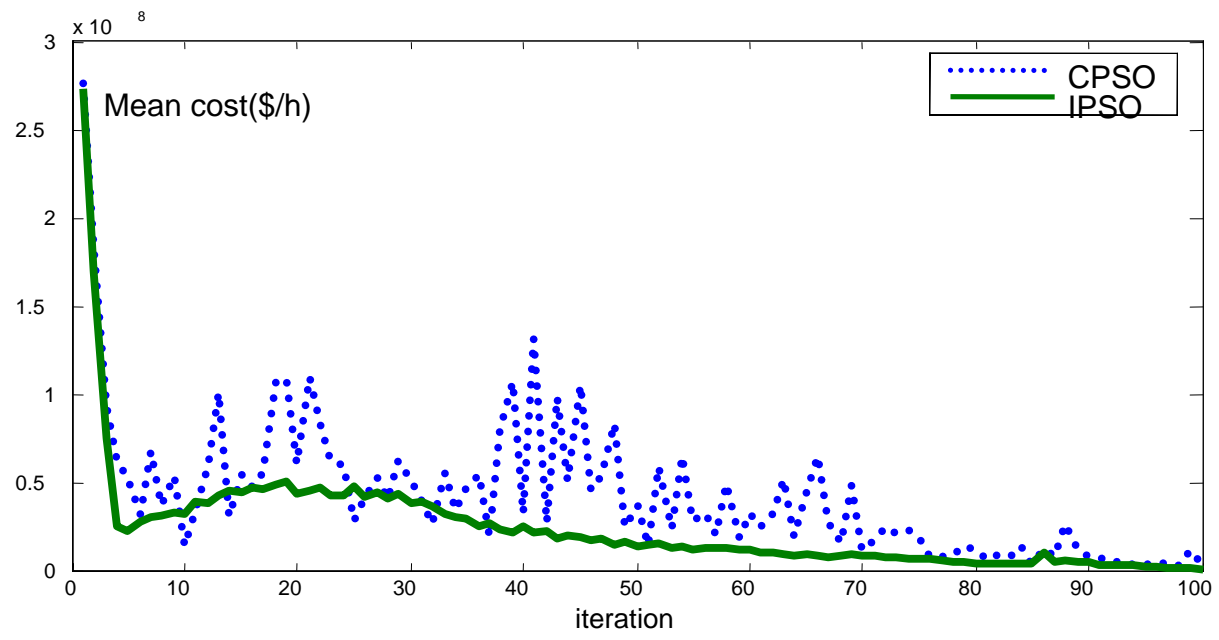

Figure 3. Variation of mean value of the swarm for CPSO and IPSO 


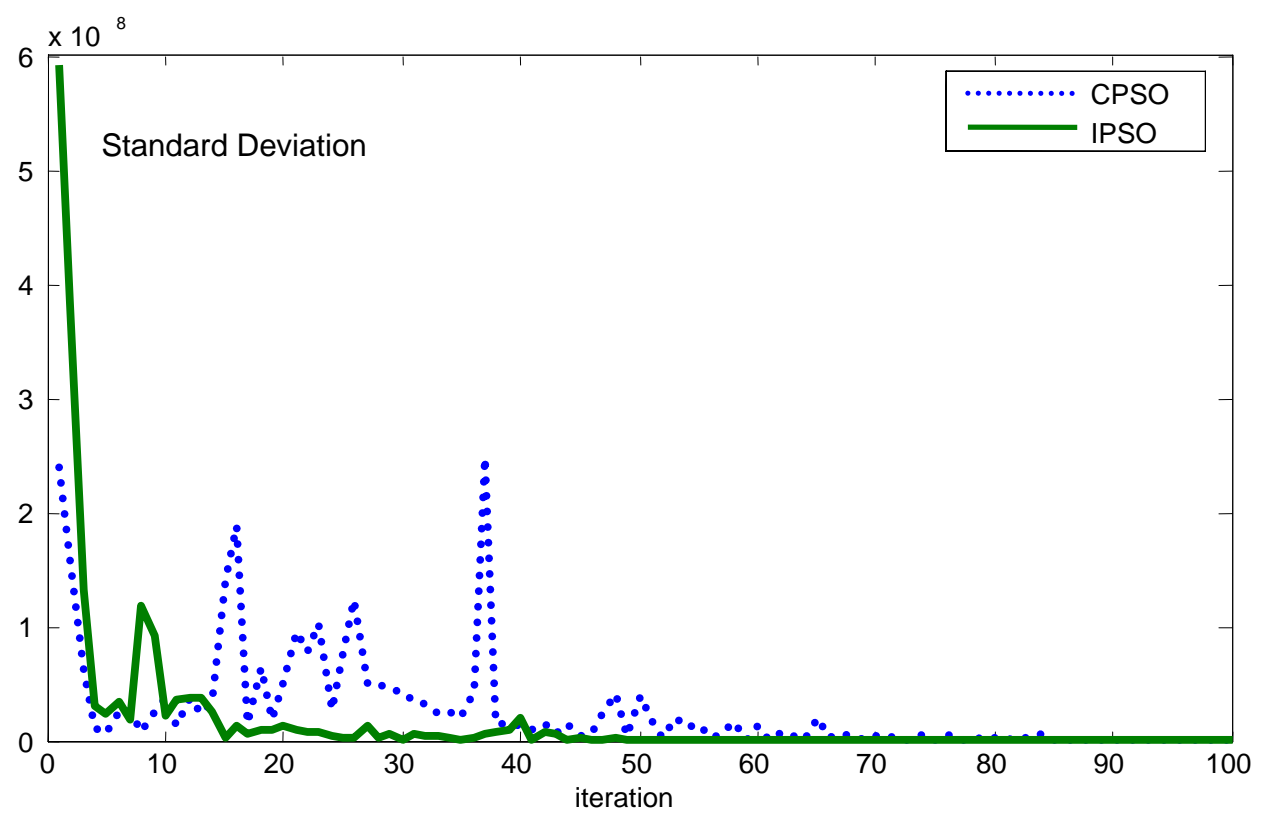

Figure 4. Variation of standard deviation of the swarm for CPSO and IPSO

Table 3. Comparison of best results of Test Case I for different loads

\begin{tabular}{|c|c|c|c|c|c|c|}
\hline \multirow{2}{*}{$\begin{array}{c}\text { Units } \\
\text { (MW) }\end{array}$} & \multicolumn{5}{|c|}{ Load Demand } \\
\cline { 2 - 7 } & \multicolumn{2}{|c|}{$300 \mathrm{MW}$} & \multicolumn{2}{c|}{$400 \mathrm{MW}$} & \multicolumn{2}{c|}{$470 \mathrm{MW}$} \\
\cline { 2 - 7 } & CPSO & IPSO & CPSO & IPSO & CPSO & IPSO \\
\hline P1 & 183.3945 & 183.9845 & 215.6883 & 221.8246 & 250.0000 & 250.0001 \\
\hline P2 & 45.8225 & 45.5391 & 91.7337 & 78.1754 & 120.0000 & 119.9999 \\
\hline P3 & 70.7830 & 70.4764 & 100.0000 & 100.0000 & 100.0000 & 100.0000 \\
\hline Cost(\$/h) & 3482.8701 & 3482.8674 & 4561.9250 & 4561.4979 & 5345.7735 & 5345.7707 \\
\hline violation & 0.0000 & 0.0000 & 0.0000 & 0.0000 & 0.0000 & 0.0000 \\
\hline & & & & & & \\
\hline
\end{tabular}

Table 4 Comparison of best results for Test Case II (with loss)

\begin{tabular}{|c|c|c|c|c|c|c|}
\hline Output(MW) & IPSO & CPSO & DE/BBO[14] & APSO[18] & GA[ 19] & TPNN[20] \\
\hline P1 & 200.5714 & 219.3163 & 207.637 & 200.528 & 194.26 & 165 \\
\hline P2 & 78.2694 & 60.0000 & 87.2833 & 78.2776 & 50 & 113.45 \\
\hline P3 & 34.0000 & 34.0000 & $15.0000^{*}$ & $33.9918^{*}$ & 79.62 & 34 \\
\hline Total power & 312.8408 & 313.3163 & 309.9203 & 312.7974 & 323.88 & 312.45 \\
\hline loss & 12.8409 & 13.3164 & 9.9204 & 12.8364 & 24.011 & 12.45 \\
\hline Violation & -0.0001 & -0.0001 & -0.0001 & -0.039 & 0.131 & 0.000 \\
\hline cost (\$/h) & $\mathbf{3 6 3 4 . 7 6 9 0}$ & 3639.6686 & 3619.7565 & 3634.3127 & 3737.20 & 3652.6000 \\
\hline Time (sec) & 0.2057 & 0.2068 & 0.015 & - & - & \\
\hline
\end{tabular}

* Solution violating ramp rate limits 
Table 5. Best results of Test Case III (with VPL) for different loads

\begin{tabular}{|c|c|c|c|c|c|c|}
\hline \multirow{2}{*}{ Units } & \multicolumn{5}{|c|}{ Load Demand } \\
\cline { 2 - 7 } & \multicolumn{2}{|c|}{$300 \mathrm{MW}$} & \multicolumn{2}{c|}{$400 \mathrm{MW}$} & \multicolumn{2}{c|}{$470 \mathrm{MW}$} \\
\cline { 2 - 7 } & CPSO & IPSO & CPSO & IPSO & CPSO & IPSO \\
\hline P1 & 184.5313 & 188.2885 & 244.7938 & 250.0000 & 250.0000 & 250.0000 \\
\hline P2 & 48.4691 & 44.7115 & 88.2060 & 50.0000 & 120.2319 & 121.8858 \\
\hline P3 & 67.0000 & 67.0000 & 67.0000 & 99.9999 & 99.7683 & 98.1141 \\
\hline Cost & 3518.0378 & $\mathbf{3 4 9 9 . 8 8 4 2}$ & 4645.2900 & $\mathbf{4 6 3 4 . 3 5 4 9}$ & 5444.1727 & $\mathbf{5 4 3 0 . 0 7 0 6}$ \\
\hline Violation & 0.0004 & 0.0001 & 0.0002 & 0.0001 & 0.0002 & 0.0000 \\
\hline
\end{tabular}

\subsection{Robustness}

The performance of heuristic search based optimization algorithms is judged through many trials with different initial populations to compare the robustness/consistency of IPSO with CPSO. The lowest cost for each of the 50 different trials has been plotted in Figure 6 for the complex Test Case III. It can be seen that IPSO method produces lowest cost most consistently as compared to the CPSO.

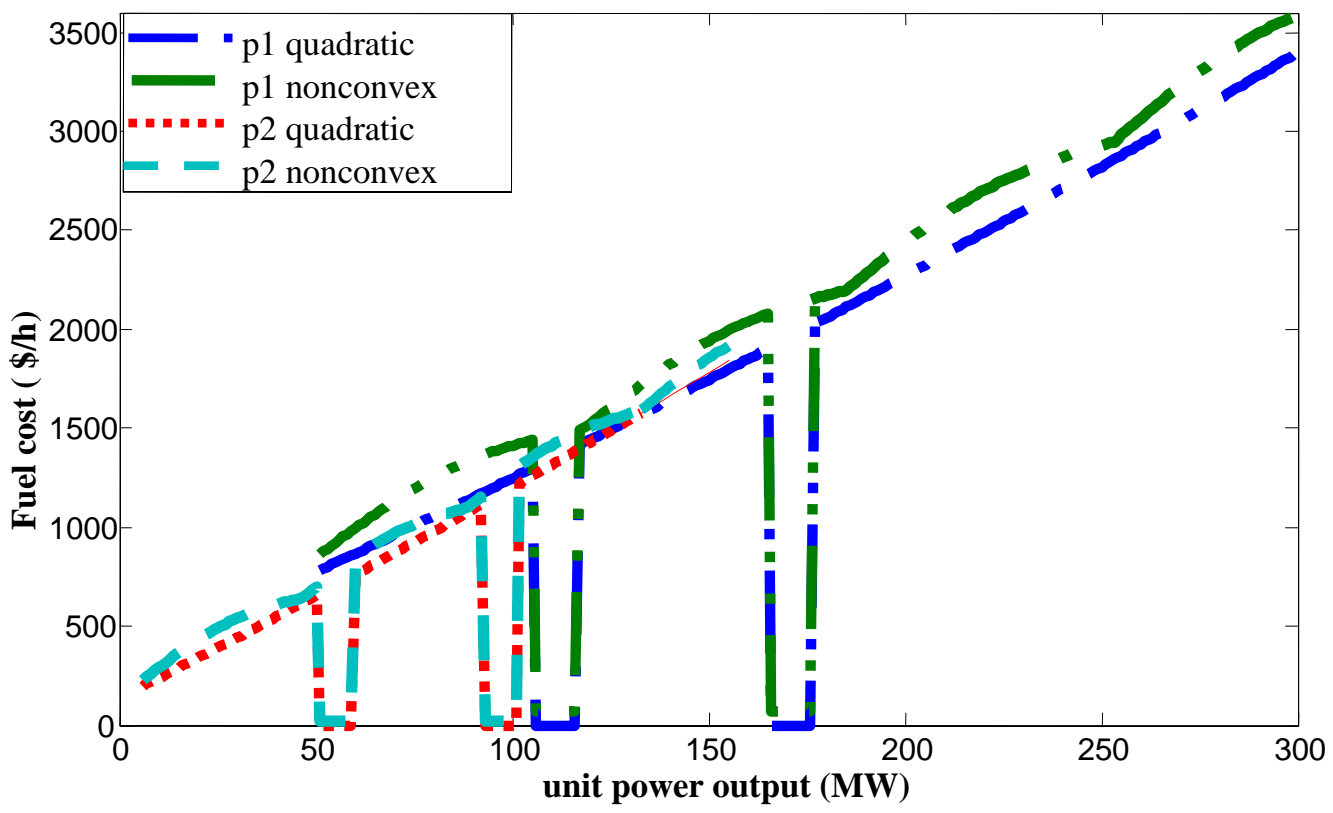

Figure 5 (a). Cost curves of generating units with and without valve point loading effects 


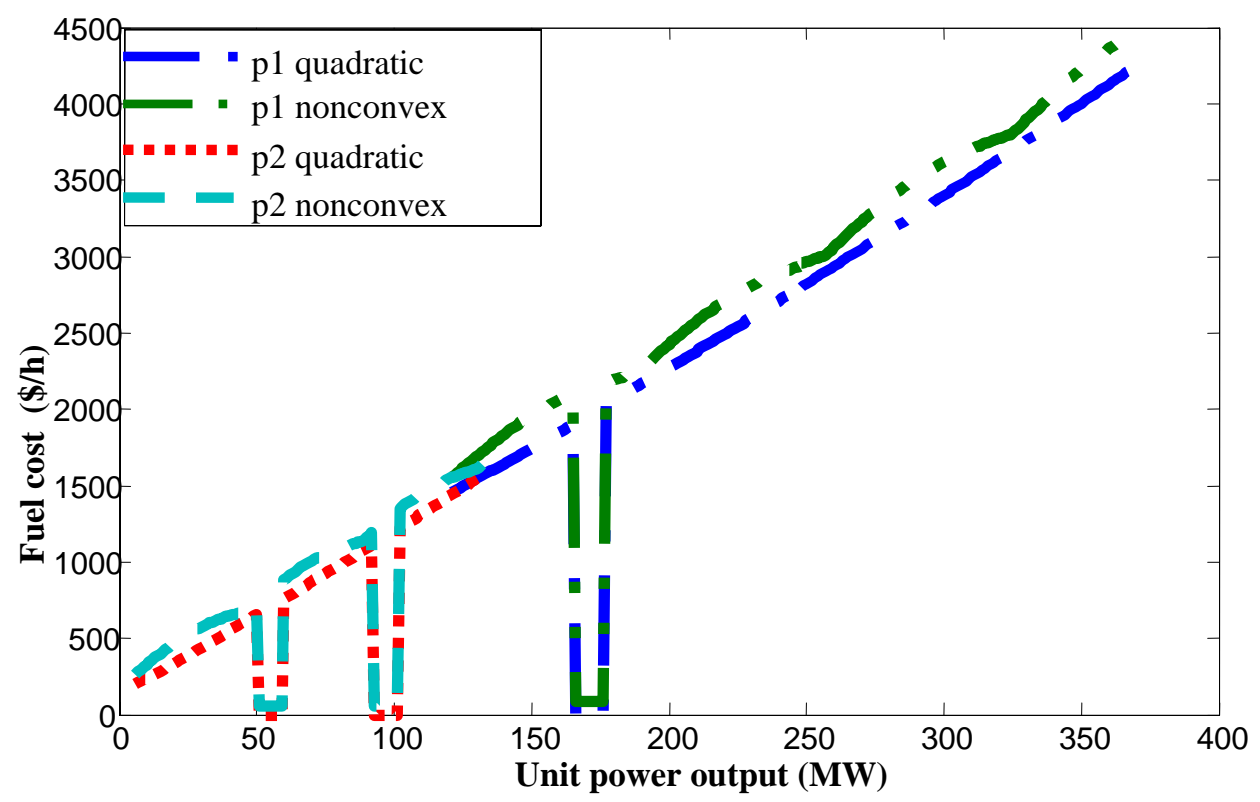

Figure 5 (b). Effect of ramp rate limits on cost curves of units with/without VPL effects

\subsection{Solution of Nonconvex Dynamic Economic Dispatch (NCDED)}

So far static dispatch results were computed for different loads and varying constraints, considering the ramp rate limits and prohibited operating zones mentioned in data Tables in the Appendix Section for one/next hour only. In NCDED the solution for the first hour is the initial solution for the next hour and optimal dispatches are computed on hourly basis for 24-hour period, for a given load. The losses have been neglected here but POZ and VPL are considered. The optimal solutions for Test Case IV for 24hours are listed in Table 6. The optimal solutions for NCDED of Test Case V with VPL are listed in Table 7. Cpu time/trial has been shown for all test cases in Table 8.

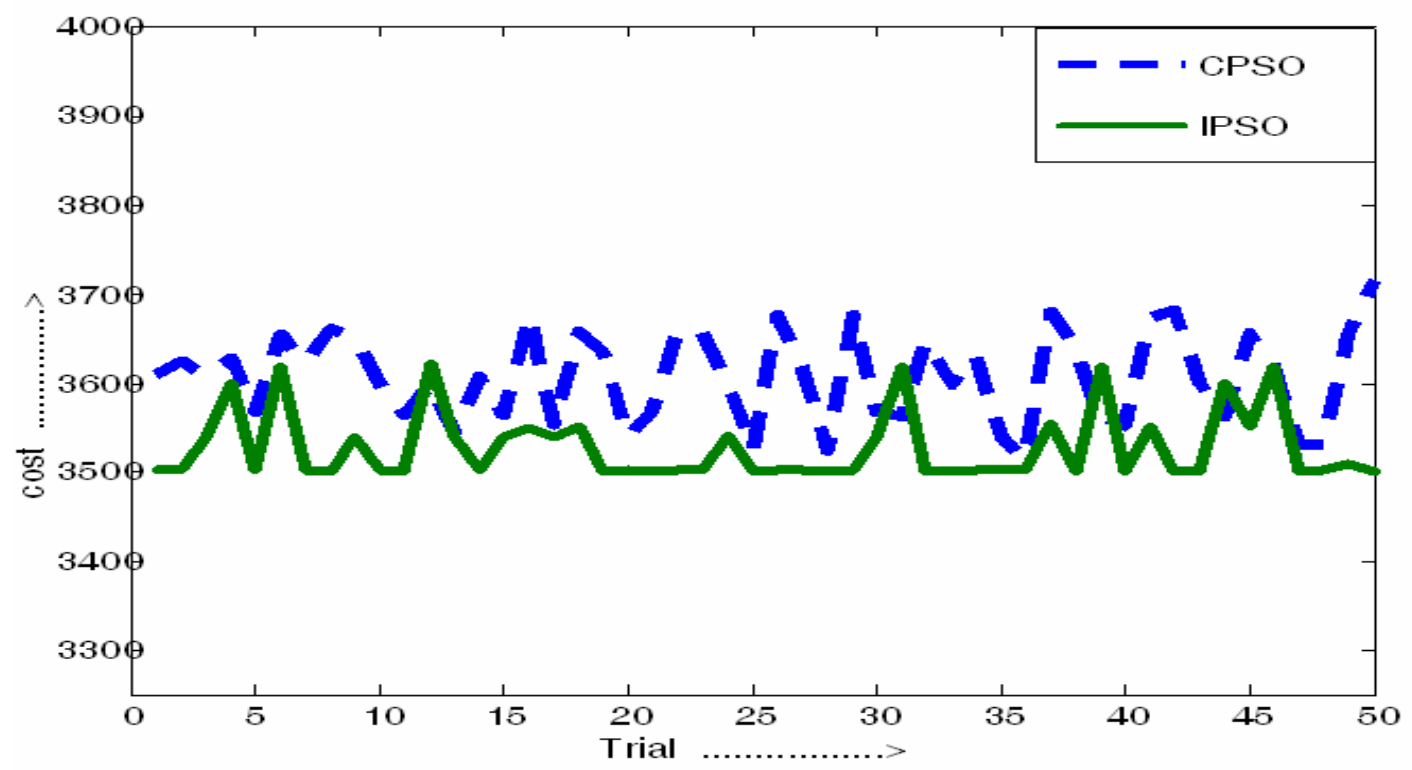

Figure 6. Comparison of best results of CPSO and IPSO (Case III) 
Table 6 Results of optimal dynamic dispatch using IPSO (Test case IV))

\begin{tabular}{|c|c|c|c|c|c|c|}
\hline Hour & Load(MW) & P1(MW) & P2(MW) & P3(MW) & Cost $(\$ / \mathrm{h})$ & Violation \\
\hline 1 & 300 & 183.9845 & 45.5391 & 70.4764 & 3482.8674 & 0.0001 \\
\hline 2 & 315 & 189.7884 & 49.9763 & 75.2352 & 3642.2181 & 0.0001 \\
\hline 3 & 330 & 197.3877 & 50.0000 & 82.6123 & 3802.6432 & 0.0001 \\
\hline 4 & 336 & 195.3137 & 60.0000 & 80.6863 & 3866.8395 & 0.0001 \\
\hline 5 & 342 & 198.5733 & 60.0000 & 83.4267 & 3931.2267 & 0.0001 \\
\hline 6 & 352 & 202.6541 & 61.9055 & 87.4403 & 4038.9542 & 0.0001 \\
\hline 7 & 361 & 206.4414 & 64.2721 & 90.2862 & 4136.2532 & 0.0002 \\
\hline 8 & 380 & 213.4426 & 70.7636 & 95.7937 & 4342.6653 & 0.0000 \\
\hline 9 & 392 & 218.4550 & 73.8838 & 99.6611 & 4473.7493 & 0.0001 \\
\hline 10 & 405 & 224.7052 & 80.2947 & 100.0000 & 4616.5297 & 0.0001 \\
\hline 11 & 445 & 242.9999 & 102.0000 & 100.0000 & 5061.9563 & 0.0001 \\
\hline 12 & 470 & 250.0000 & 119.9999 & 100.0000 & 5345.7707 & 0.0001 \\
\hline 13 & 400 & 223.7784 & 77.8661 & 98.3556 & 4561.6153 & 0.0001 \\
\hline 14 & 382 & 213.5666 & 71.5456 & 96.8878 & 4364.4719 & 0.0001 \\
\hline 15 & 370 & 209.5917 & 66.9317 & $93.4766]$ & 4233.8547 & 0.0000 \\
\hline 16 & 364 & 207.0180 & 65.7036 & 91.2782 & 4168.7511 & 0.0002 \\
\hline 17 & 355 & 203.7440 & 63.1887 & 88.0672 & 4071.3522 & 0.0000 \\
\hline 18 & 345 & 200.3401 & 60.0000 & 84.6598 & 3963.4960 & 0.0001 \\
\hline 19 & 339 & 196.5646 & 60.0000 & 82.4353 & 3899.0099 & 0.0000 \\
\hline 20 & 325 & 195.1397 & 50.0000 & 79.8602 & 3749.0297 & 0.0001 \\
\hline 21 & 320 & 192.1366 & 50.0000 & 77.8634 & 3695.5536 & 0.0001 \\
\hline 22 & 316 & 189.8123 & 50.0000 & 76.1877 & 3652.8744 & 0.0001 \\
\hline 23 & 310 & 187.4466 & 48.5125 & 74.0409 & 3589.0058 & 0.0001 \\
\hline 24 & 300 & 183.8532 & 45.3336 & 70.8131 & 3482.8684 & 0.0000 \\
\hline
\end{tabular}

Table 7 Results of optimal dynamic dispatch using IPSO (Test case V)

\begin{tabular}{|c|c|c|c|c|c|c|}
\hline Hour & Load(MW) & P1(MW) & P2(MW) & P3(MW) & Cost $(\$ / h)$ & violation \\
\hline 1 & 300 & 187.5215 & 45.4785 & 67.0000 & 3499.9827 & 0.0000 \\
\hline 2 & 315 & 229.1125 & 5.0000 & 80.8874 & 3671.7802 & 0.0001 \\
\hline 3 & 330 & 202.4080 & 46.8879 & 80.7041 & 3804.5780 & 0.0000 \\
\hline 4 & 336 & 240.4247 & 46.8879 & 48.6874 & 3904.6568 & 0.0000 \\
\hline 5 & 342 & 213.7202 & 46.8883 & 81.3913 & 3944.2733 & 0.0001 \\
\hline 6 & 352 & 187.0157 & 88.7758 & 76.2085 & 4070.2219 & 0.0000 \\
\hline 7 & 361 & 228.60671 & 50.0000 & 82.3932 & 4171.2388 & 0.0001 \\
\hline 8 & 380 & 201.9022 & 88.7758 & 89.3219 & 4376.5764 & 0.0001 \\
\hline 9 & 392 & 243.4930 & 91.1328 & 57.3742 & 4508.9031 & 0.0000 \\
\hline 10 & 405 & 216.7885 & 138.7965 & 49.4149 & 4668.0087 & 0.0001 \\
\hline 11 & 445 & 225.4112 & 140.4746 & 79.1141 & 5131.1313 & 0.0000 \\
\hline 12 & 470 & 223.2955 & 150.0000 & 96.7044 & 5384.8987 & 0.0001 \\
\hline 13 & 400 & 196.5910 & 113.8879 & 89.5211 & 4606.5498 & 0.0000 \\
\hline 14 & 382 & 237.2292 & 77.7708 & 67.0000 & 4418.3082 & 0.0001 \\
\hline 15 & 370 & 210.5247 & 88.7758 & 70.6995 & 4276.2967 & 0.0000 \\
\hline 16 & 364 & 183.8202 & 102.0000 & 78.1798 & 4225.0135 & 0.0000 \\
\hline 17 & 355 & 157.1157 & 107.7758 & 90.1084 & 4139.2634 & 0.0001 \\
\hline 18 & 345 & 198.7067 & 71.6637 & 74.6296 & 4014.9798 & 0.0001 \\
\hline 19 & 339 & 240.2977 & 46.8879 & 51.8143 & 3937.2702 & 0.0001 \\
\hline 20 & 325 & 213.5932 & 88.7758 & 22.6309 & 3811.2918 & 0.0001 \\
\hline 21 & 320 & 186.8887 & 86.0543 & 47.0570 & 3752.6818 & 0.0000 \\
\hline 22 & 316 & 228.4797 & 8.0543 & 79.4659 & 3673.5408 & 0.0001 \\
\hline 23 & 310 & 201.7752 & 48.2247 & 60.0000 & 3645.6840 & 0.0001 \\
\hline 24 & 300 & 177.0000 & 46.8879 & 76.1121 & 3508.8980 & 0.0000 \\
\hline
\end{tabular}


Table 8 CPU time for the different test cases

\begin{tabular}{|c|c|c|c|c|c|}
\hline TEST CASE & Test case I & Test case II & Test case III & Test case IV & Test case V \\
\hline cpu time/trial & 0.1412 & 0.2057 & 0.1562 & 3.4129 & 3.7613 \\
\hline
\end{tabular}

\section{Conclusions}

An improved PSO based strategy is proposed in this paper for solving the nonconvex dynamic economic dispatch (NCDED problem. The proposed IPSO strategy is found to improve the performance of PSO and to handle the problem of premature convergence found in CPSO very effectively by i)generating crazy particles whose velocities are reinitialized with a certain probability ii) employing iterative variation of cognitive and social parameters. The superiority of IPSO becomes more evident for more complex systems having multiple minima. This method outperforms CPSO in terms of solution quality, computational efficiency, dynamic convergence, robustness and stability. The proposed IPSO approach is tested on five different test cases having different levels of complexities and constraints. The results are compared with previously published results and found to be superior or/and comparable. The IPSO is capable of handling all the complex constraints imposed by ramp rate limits, valve point loading effects and prohibited operating zones very effectively.

\section{Nomenclature}

$P_{i} \quad$ Power output of the $i^{\text {th }}$ generating unit

$F_{i}\left(P_{i}\right) \quad$ Fuel cost function of the $i^{\text {th }}$ generating unit

$P_{i}^{\min }, P_{i}^{\max }$ Minimum and maximum generation limits on $\mathrm{i}^{\text {th }}$ unit

$N \quad$ Number of generating units

$\mathrm{P}_{\mathrm{D}} \quad$ Total real power demand

$\mathrm{P}_{\mathrm{L}} \quad$ Total real power loss

$D R_{i} \quad$ Down ramp rate limit

$U R_{i} \quad$ Up ramp rate limit

$x_{i d} \quad$ position of $d^{\text {th }}$ dimension of the $i^{\text {th }}$ particle

$v_{\text {id }} \quad$ velocity of $d^{\text {th }}$ dimension of the $i^{\text {th }}$ particle

\section{Appendix}

Table A1. Cost coefficients and unit operating limits

\begin{tabular}{|c|c|c|c|c|c|c|c|}
\hline unit & $P_{\min }$ & $P_{\max }$ & $a_{i}\left(\$ / \mathrm{MW}^{2}\right)$ & $b_{i}(\$ / \mathrm{MW})$ & $c_{l}(\$)$ & $e_{i}$ & $f_{i}$ \\
\hline 1 & 50 & 250 & 0.00525 & 8.663 & 328.13 & 125 & 0.046 \\
\hline 2 & 5 & 150 & 0.00609 & 10.04 & 136.91 & 75 & 0.075 \\
\hline 3 & 15 & 100 & 0.00592 & 9.76 & 59.16 & 50 & 0.098 \\
\hline
\end{tabular}

Table A2. Ramp rate limits and prohibited operating zones

\begin{tabular}{|c|c|c|c|c|}
\hline unit & $P_{t}^{\ell}$ & $U R_{t}(\mathrm{MW} / \mathrm{h})$ & $D R_{i}(\mathrm{MW} / \mathrm{h})$ & Prohibited zone $(\mathrm{MW})$ \\
\hline 1 & 215 & 55.0 & 97.0 & {$[105,117][165,177]$} \\
\hline 2 & 72.0 & 55.0 & 78.0 & {$[50,60][92,102]$} \\
\hline 3 & 98.0 & 45.0 & 64.0 & {$[25,32][60,67]$} \\
\hline
\end{tabular}


Table A3. B-loss coefficients of three unit systems

\begin{tabular}{|c|c|c|c|}
\hline \multirow{3}{*}{ Bij } & 0.000136 & 0.0000175 & 0.000184 \\
\cline { 2 - 4 } & 0.0000175 & 0.000154 & 0.000283 \\
\cline { 2 - 4 } & 0.000184 & 0.000283 & 0.00165 \\
\hline
\end{tabular}

\section{Acknowledgement}

The authors sincerely acknowledge the financial support provided by UGC under major research project entitled Power System Optimization and Security Assessment Using Soft Computing Techniques, vide F No.34-399/2008 (SR) dated, 24th December 2008. The authors also thank the Director, M.I.T.S. Gwalior for providing facilities for carrying out this work. The third author acknowledges UGC research award for post doctoral work sanctioned by UGC, New Delhi vide letter no. F-30-120(SC)/2009 (SA-II).

\section{References}

Bhattacharya A. and Chattopadhyay P.K., 2010.Hybrid differential evolution with biogeography- based optimization for solution of economic load dispatch. IEEE Transactions on Power System, Vol. 25, No.4, pp. 1955 - 1964.

Chaturvedi K. T., Pandit M. and Srivastava L., 2008. Self-organizing hierarchical particle swarm optimization for nonconvex economic dispatch. IEEE. Transactions on Power System, Vol. 23, No. 3, pp. 1079-1087

Chen P.H. and Chang H.C., 1995. Large scale economic dispatch approach by genetic algorithm. IEEE Transactions on Power Systems, Vol. 10, No.4, pp. 1919-1926,.

Chiou J. P., 2009. A variable scaling hybrid differential evolution for solving large-scale power dispatch problems. IET Generation, Transmission Distribution, Vol. 3, No. 2, pp. 154-163.

Damousis Ioannis G., Bakirtzis Anastasios G. and Dokopolous Petros S., 2003. Network constrained economic dispatch using realcoded genetic algorithms. IEEE Transactions on Power System, Vol. 18, No. 1, pp. 198-205.

Kennedy J. and Eberhart R., 1995.Particle swarm optimization. in Proc. IEEE Conf. on Neural Networks (ICNN'95), Vol. IV, Perth, Australia, pp.1942-1948.

Naresh R., Dubey J. and Sharma J.D., 2004. Two-phase neural network based frame work for modelling of constrained economic load dispatch. IEE proceedings Generation, Transmission and Distribution, Vol. 151, No. 3, pp. 373 -380.

Orero S. O.and Irving M.R., 1996. Economic dispatch of generators with prohibited operating zones: a genetic algorithm approach. IEE proceedings, Generation, Transmission and Distribution, Vol. 143, No. 6

Panigrahi B. K., Pandi V. R., and Das S., 2008. Adaptive particle swarm optimization approach for static and dynamic economic load dispatch. Energy Conversion and Management, Vol. 49, No. 6, pp. 1407-1415.

Park J. B. Jeong Y.W, Shin J.R.and Lee K.Y., 2007. An improved particle swarm optimization for nonconvex economic dispatch problems, IEEE. Transactions on Power system, Vol. 25, No. 1, pp.156-166.

Park J. B., Jeong Y.W. and Shin J.R., 2010. An improved particle swarm optimization for nonconvex economic dispatch problems. IEEE Transactions on Power System, Vol. 25, No. 1, pp.156-166.

Ratnaweera A., Halgamuge S.K. and Watson H.C., 2004 Self-organizing hierarchical Particle swarm optimizer with time-varying acceleration coefficients, IEEE Transactions on Evolutionary Computation, Vol. 8, No. 3, pp.240-255

Selvakumar A. I. and Thanushkodi K., 2007. A new particle swarm optimization solution to nonconvex economic dispatch problems. IEEE. Transactions on Power System, Vol. 22, No. 1, pp.42-51.

Shi Y. and Eberhart R.C., 1999. Empirical study of particle swarm optimization, Proc. of IEEE Int. Congress on Evolutionary Computation, Vol. 3, pp. 101-106.

Shoults R.R., 1986. A dynamic programming based method for developing dispatch curves when incremental heat rate curves are non-monotonically increasing. IEEE Transactions on Power System, Vol. 1, pp.10-16.

Sinha N., Chakrabarti R. and Chattopadhyay P.K., 2003. Evolutionary programming techniques for economic load dispatch", IEEE Transactions on Evolutionary Computation, Vol. 7, No. 1, pp. 83-94.

Victoire T.A.A. and Jeyakumar A.E., 2005. Reserve constrained dynamic dispatch of units with valve point effects. IEEE Transactions on Power System, Vol. 20, No. 3, pp. 1273-1282.

Walter D.C. and Sheble G. B., 1993. Genetic algorithm solution of economic load dispatch with valve point loading. IEEE Transactions on Power System, Vol. 8, pp.1325-1332.

Wang and Shahidehpour S.M., 1993. Effects of ramp-rate limits on unit commitment and economic dispatch. IEEE Transactions on Power System, Vol. 8, No. 3, pp.1341-1350

Wood A.J. and Wollenberg B.F., 1984. Power Generation Operation and Control, New York: Wiley. 


\section{Biographical notes}

Rajkumari Batham obtained her B.E. degree in Electrical Engineering from M.I.T.S., Gwalior, (India) in 2008 and is presently persuing M.Tech in Industrial systems and Drives from M.I.T.S., Gwalior.

Kalpana Jain obtained her M.E. degree in Electrical Engineering from Madhav Institute of Technology \& Science Gwalior (India) in 2010. She is currently working as Project Fellow in the department of Electrical Engineering, M.I.T.S., Gwalior (India). Her areas of interest are Power System Security Analysis, Optimization and soft computing/evolutionary methods.

Manjaree Pandit obtained her M.Tech degree in Electrical Engineering from Maulana Azad College of Technology, Bhopal, (India) in 1989 and Ph.D. degree from Jiwaji University Gwalior (India) in 2001. She is currently working as Professor in Department of Electrical Engineering, M.I.T.S., Gwalior, (India). Her areas of interest are Power System Security Analysis, Optimization using soft computing/ evolutionary methods, ANN and Fuzzy neural applications to Power System.

Received June 2011

Accepted June 2011

Final acceptance in revised form June 2011 